\title{
The global parametrix in the Riemann-Hilbert steepest descent analysis for orthogonal polynomials
}

\author{
Arno Kuijlaars* and Man Yue $\mathrm{Mo}^{\dagger}$
}

\begin{abstract}
In the application of the Deift-Zhou steepest descent method to the Riemann-Hilbert problem for orthogonal polynomials, a model Riemann-Hilbert problem that appears in the multi-cut case is solved with the use of hyperelliptic theta functions. We present here an alternative approach which uses meromorphic differentials instead of theta functions to construct the solution of the model Riemann-Hilbert problem. By using this representation, we obtain a new and elementary proof for the solvability of the model Riemann-Hilbert problem.
\end{abstract}

\section{The global parametrix}

\section{$1.1 \quad$ Introduction}

The Deift-Zhou steepest descent method is a powerful technique in the asymptotic analysis of Riemann-Hilbert problems that has been successfully applied to numerous problems in integrable systems, random matrix theory

*Department of Mathematics, Katholieke Universiteit Leuven, Celestijnenlaan 200B, B-3001 Leuven, Belgium. email: arno.kuijlaars@wis.kuleuven.be. The work of the first author is supported by FWO-Flanders project G.0427.09, by K.U. Leuven research grant OT/08/33, by the Belgian Interuniversity Attraction Pole P06/02, by the European Science Foundation Program MISGAM, and by grant MTM2008-06689-C02-01 of the Spanish Ministry of Science and Innovation.

${ }^{\dagger}$ Department of Mathematics, University of Bristol, Bristol BS8 1TW, U.K. email: m.mo@bristol.ac.uk. The second author is supported by the EPSRC grant EP/G019843/1. 
and orthogonal polynomials, see e.g. [5], 6], [7, [8], [16]. When applying the steepest descent method as in [7, one performs a series of transformations to the $2 \times 2$ matrix-valued Riemann-Hilbert problem for orthogonal polynomials to eventually approximate it by a "model Riemann-Hilbert problem" which is also known as the "global parametrix" or the "outer parametrix". The solution of the model Riemann-Hilbert $(\mathrm{RH})$ problem in [1], [6], [7, 9], [16, Section 4.3] and [17] uses Riemann theta functions on hyperelliptic Riemann surfaces. In [19], such approach was generalized to higher dimensional Riemann-Hilbert problems with quasi-permutation jump matrices and the solutions were expressed in terms of Riemann theta functions together with the Szegö kernel. These constructions use various notions from Riemann surfaces, which, although classical, require a fair amount of background in algebraic geometry.

In this paper, we present an alternative approach to the solution of the model RH problem. The approach in this paper is less constructive since it does not lead to explicit formulas. However, in many applications, such as the universality results of random matrix theory, one is merely interested in the existence of a global parametrix, rather than its explicit form. In such cases, the lack of explicit formulas is not an issue. The present approach also enables us to obtain a new elementary proof for the solvability of the model Riemann-Hilbert problem.

On the other hand, our approach is conceptually rather simple (in our opinion), and generalizes without too much effort from hyperelliptic (i.e., twosheeted) Riemann surfaces to multi-sheeted Riemann surfaces which arise in the steepest descent analysis of larger size RH problems associated with multiple orthogonal polynomials [22]. In [11], the RH steepest descent analysis of a 4 x 4 matrix-valued Riemann-Hilbert problem was done with the help of an associated Riemann surface that is a four-sheeted cover of the Riemann sphere. The analysis in [11] was restricted to the one-cut case (i.e., genus zero). The extension to the multi-cut case was done in [21] where both meromorphic differentials and Riemann theta functions are used to solve the model RH problem. Analyzing the approach in [21] we found that it is also possible to avoid the use of Riemann theta functions completely and to use meromorphic differentials only. For the sake of clarity we present this approach here for the case of the $2 \times 2$ matrix-valued model $\mathrm{RH}$ problem as it arises in the steepest descent analysis for orthogonal polynomials. We will use this construction in the forthcoming work [12] for a $4 \times 4$ matrix-valued $\mathrm{RH}$ problem. See also 2 for a similar situation in a $3 \times 3$ context. 
One of the main problems in solving the model Riemann-Hilbert problem is the proof of its solvability. As pointed out in [9] and [17, the model Riemann-Hilbert problem can be represented as the monodromy problem of the Schlesinger equation. To see whether the monodromy problem is solvable, one can construct an isomonodromic tau function [15], [20], corresponding to the Schlesinger equation and the monodromy problem will be solvable if the value of the tau function is non-zero. However, the determination of the zeroes of the tau function, known as the Malgrange divisor, is often a difficult task. In [17] and [19], it was shown that for the type of model RiemannHilbert problem considered here, the isomonodromic tau function is zero if and only if a certain theta function is zero. Therefore to prove the solvability of the model Riemann-Hilbert problem, one would need to study the theta divisor, which is a highly transcendental object. By using the approach in this paper, we were able to use much more elementary arguments to show the existence of the global parametrix in the hyperelliptic case, which allows us to avoid the theta divisor completely.

\subsection{The model Riemann-Hilbert problem}

The model RH problem that arises in the application of the Deift-Zhou steepest descent method to orthogonal polynomials is the following.

We are given $N$ intervals $\left[a_{k}, b_{k}\right], k=1, \ldots, N$ on the real line ordered so that $b_{k}<a_{k+1}$ for $k=1, \ldots, N-1$. We also have $N-1$ real numbers $\alpha_{k}$ for $k=1, \ldots, N-1$, and an integer $n$. The aim is then to construct a solution of the following $\mathrm{RH}$ problem.

Definition 1.1. The model Riemann-Hilbert problem is the following $\mathrm{RH}$ problem for a $2 \times 2$-matrix valued function $M: \mathbb{C} \backslash\left[a_{1}, b_{N}\right] \rightarrow \mathbb{C}^{2 \times 2}$ :

(a) $M$ is analytic on $\mathbb{C} \backslash\left[a_{1}, b_{N}\right]$,

(b) $M$ has jumps $M_{+}(x)=M_{-}(x) J_{M}(x)$ for $x \in\left[a_{1}, b_{N}\right]$ where

$$
J_{M}(x)=\left(\begin{array}{cc}
0 & 1 \\
-1 & 0
\end{array}\right), \quad \text { for } x \in\left(a_{k}, b_{k}\right),
$$

for $k=1, \ldots, N$,

$$
J_{M}(x)=\left(\begin{array}{cc}
e^{-2 \pi i n \alpha_{k}} & 0 \\
0 & e^{2 \pi i n \alpha_{k}}
\end{array}\right), \quad \text { for } x \in\left(b_{k}, a_{k+1}\right),
$$

for $k=1, \ldots, N-1$, 
(c) $M(z)=I+O(1 / z)$ as $z \rightarrow \infty$,

(d) $M$ has at most fourth-root singularities near the endpoints $a_{k}$ and $b_{k}$.

Clearly the jump condition (1.2) in the model RH problem only depends on the value of the numbers $n \alpha_{k}$ modulo the integers, and so we may (and usually do) consider them to belong to $\mathbb{R} / \mathbb{Z}$.

The model RH problem was stated and solved in [7]. The goal of this paper is to present an alternative construction and to show that away from the endpoints $a_{k}$ and $b_{k}$, the solution and its inverse are bounded in $n$.

As already mentioned, we use the hyperelliptic Riemann surface with cuts along the intervals $\left[a_{k}, b_{k}\right]$. The main role is played by the meromorphic differentials $\omega_{P}^{(\nu)}, \nu=1,2$, introduced in Definition 2.1. The meromorphic differential depends on $N-1$ points $P_{1}, \ldots, P_{N-1}$ on the Riemann surface. The heart of the matter is Theorem 2.6 which states that a suitably defined mapping from $P_{1}, \ldots, P_{N-1}$ to a vector of $B$-periods is bijective. This result allows us to take the points so that the $B$-periods are exactly the numbers $2 \pi i n \alpha_{k}, k=1, \ldots, N-1$, that appear in the jump condition (1.2). In Section 3 we define the corresponding Abelian integrals, which after exponentation lead to functions $v_{j}^{(\nu)}, j, \nu=1,2$, that are used in the Definition 3.7 of the solution of the model $\mathrm{RH}$ problem.

Some further properties of $M$ are discussed in Section 4, including the fact that $M$ and $M^{-1}$ are uniformly bounded in $n$ if we stay away from the endpoints $a_{k}$ and $b_{k}$. In the final Section 5 we present an alternative construction for the second row of $M$, assuming that we know the first row of $M$.

\section{Meromorphic differentials}

The construction will be based on meromorphic differentials (Abelian differentials of the third kind) on the two-sheeted Riemann surface $\mathcal{R}$ for the equation

$$
w^{2}=\prod_{k=1}^{N}\left(z-a_{k}\right)\left(z-b_{k}\right)
$$

which is obtained by gluing together two copies of $\overline{\mathbb{C}} \backslash \bigcup_{k=1}^{N}\left[a_{k}, b_{k}\right]$ along the cuts $\left[a_{k}, b_{k}\right]$ in the usual crosswise manner. The surface is compact (we add a point at infinity to each sheet) and has genus $N-1$. 


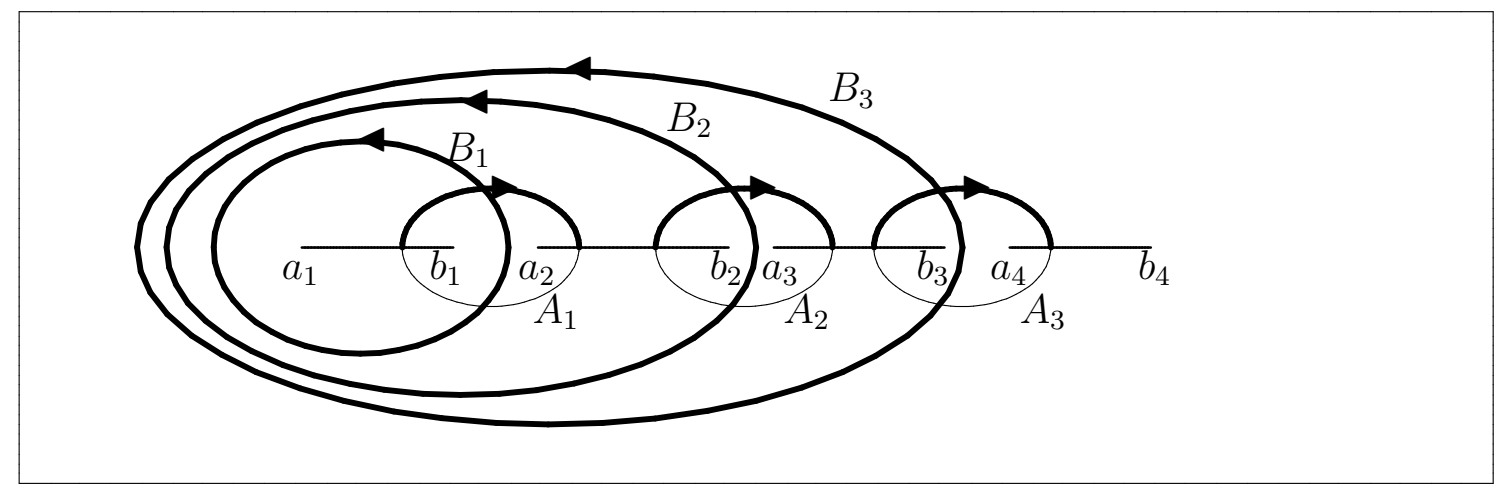

Figure 1: The canonical homology basis $\left(A_{1}, \ldots, A_{N-1}, B_{1}, \ldots, B_{N-1}\right)$ for the case $N=4$. The thick contours are on the first sheet and the thin contours are on the second sheet of the Riemann surface $\mathcal{R}$.

We need a few standard facts about Riemann surfaces. Our main reference is 13 .

We will now define the canonical homology basis on this Riemann surface. We choose the cycles $A_{j}$ and $B_{j}$ for a canonical homology basis

$$
\left(A_{1}, \ldots, A_{N-1} ; B_{1}, \ldots, B_{N-1}\right)
$$

as indicated in Figure 1 for the case $N=4$. That is, $B_{j}$ is a cycle on the first sheet that encircles the interval $\left[a_{1}, b_{j}\right]$ once in the counterclockwise direction. We choose $B_{j}$ to be symmetric with respect to the real axis on the first sheet. The cycle $A_{j}$ has a part in the upper half-plane of the first sheet, a part in the lower half-plane of the second sheet, and passes through the cuts $\left[a_{j}, b_{j}\right]$ and $\left[a_{j+1}, b_{j+1}\right]$ with an orientation also indicated in Figure 1 .

There is an anti-holomorphic involution $\phi$ on $\mathcal{R}$ which maps $z$ to $\bar{z}$ on the same sheet. The set of fixed points of $\phi$ are the real ovals, which are $N$ closed contours $\Gamma_{j}, j=0, \ldots, N-1$, where for $j=1, \ldots, N-1, \Gamma_{j}$ is the union of the two intervals $\left[b_{j}, a_{j+1}\right]$ from both sheets and $\Gamma_{0}$ is unbounded and contains the intervals from $a_{1}$ and $b_{N}$ to the two points at infinity. Thus

$$
\Gamma_{j}=\left\{(z, w) \in \mathcal{R} \mid b_{j} \leq z \leq a_{j+1}\right\}, \quad j=1, \ldots, N-1 .
$$

The cycle $A_{j}$ is homotopic to $\Gamma_{j}$ but we choose $A_{j}$ to be disjoint from $\Gamma_{j}$.

For each $j=1, \ldots, N-1$ we choose a point $P_{j} \in \Gamma_{j}$. We are going to associate with the $N-1$ points $\left(P_{1}, \ldots, P_{N-1}\right)$ and an index $\nu \in\{1,2\}$ a meromorphic differential $\omega=\omega_{P}^{(\nu)}$. 
Definition 2.1. The meromorphic differential $\omega=\omega_{P}^{(\nu)}$ associated with $\left(P_{1}, \ldots, P_{N-1}\right) \in$ $\Gamma_{1} \times \cdots \times \Gamma_{N-1}$ and $\nu \in\{1,2\}$, is defined by the following properties:

- $\omega_{P}^{(\nu)}$ has simple poles at the points $a_{1}, b_{1}, \ldots, a_{N}, b_{N}, P_{1}, \ldots, P_{N-1}$ with residues

$$
\begin{aligned}
& \operatorname{Res}\left(\omega_{P}^{(\nu)}, a_{j}\right)=\operatorname{Res}\left(\omega_{P}^{(\nu)}, b_{j}\right)=-\frac{1}{2}, \quad j=1, \ldots, N, \\
& \operatorname{Res}\left(\omega_{P}^{(\nu)}, P_{j}\right)=1, \quad j=1, \ldots, N-1,
\end{aligned}
$$

- $\omega_{P}^{(1)}$ has a simple pole at $\infty_{2}$ (the point at infinity on the second sheet) and $\omega_{P}^{(2)}$ has a simple pole at $\infty_{1}$ (the point at infinity on the first sheet) with residue

$$
\operatorname{Res}\left(\omega_{P}^{(1)}, \infty_{2}\right)=\operatorname{Res}\left(\omega_{P}^{(2)}, \infty_{1}\right)=1 .
$$

- The differential $\omega_{P}^{(\nu)}$ is holomorphic elsewhere.

- The $A$-periods satisfy:

$$
\int_{A_{j}} \omega_{P}^{(\nu)}=0, \quad j=1, \ldots, N-1 .
$$

Note that the points $P_{j}$ are not on any of the $A$-cylces.

The meromorphic differential $\omega_{P}^{(\nu)}$ exists and is uniquely defined by the properties (2.3)-(2.6). Indeed, a simple count shows that the sum of the residues in (2.3)-(2.5) is equal to zero which is a necessary and sufficient condition for the meromorphic differential to exist, see for example Theorem II.5.3 in [13]. The residue conditions determine the meromorphic differential up to a holomorphic differential. The vector space of holomorphic differentials has dimension $N-1$, and the $N-1$ conditions in (2.6) determine the meromorphic differential uniquely.

If one or more of the $P_{j}$ 's coincide with a branch point, then the residue conditions (2.3) $-(2.4)$ have to be modified appropriately. For example, if $P_{j}=b_{j}$ then

$$
\operatorname{Res}\left(\omega_{P}^{(\nu)}, P_{j}\right)=\frac{1}{2} .
$$

In this way, the meromorphic differential $\omega_{P}^{(\nu)}$ depends continuously on the $P_{j}$ 's. This fact will play a role in the proof of Proposition 2.3 below. 
The anti-holomorphic involution $\phi$ can be used to map $\omega_{P}^{(\nu)}$ to a meromorphic differential $\phi^{\#}\left(\omega_{P}^{(\nu)}\right)$ in an obvious way. If $\omega_{P}^{(\nu)}$ is equal to $f_{j}(z) d z$ for some meromorphic function $f_{j}$ on sheet $j$ for $j=1,2$, then $\phi^{\#}\left(\omega_{P}^{(\nu)}\right)$ is equal to

$$
\overline{f_{j}(\bar{z})} d z
$$

on sheet $j$. A crucial property is that $\omega_{P}^{(\nu)}$ is invariant under the map $\phi^{\# \text {. }}$

Lemma 2.2. For every $\left(P_{1}, \ldots, P_{N-1}\right) \in \Gamma_{1} \times \cdots \times \Gamma_{N-1}$ and $\nu \in\{1,2\}$, we have

$$
\omega_{P}^{(\nu)}=\phi^{\#}\left(\omega_{P}^{(\nu)}\right)
$$

Proof. Since all poles of $\omega_{P}^{(\nu)}$ are invariant under $\phi$, the meromorphic differential $\phi^{\#}\left(\omega_{P}^{(\nu)}\right)$ has the same (simple) poles as $\omega_{P}^{(\nu)}$ with the same residues. For the $A$-periods we have by definition of $\phi$,

$$
\int_{A_{j}} \phi^{\#}\left(\omega_{P}^{(\nu)}\right)=\overline{\int_{\phi\left(A_{j}\right)} \omega_{P}^{(\nu)}}, \quad j=1, \ldots, N-1 .
$$

The cycle $\phi\left(A_{j}\right)$ is homotopic to $A_{j}$ in $\mathcal{R}$. In the process of deforming $\phi\left(A_{j}\right)$ to $A_{j}$ we pick up residue contributions from the poles of $\omega_{P}^{(\nu)}$ at $b_{j}, P_{j}$ and $a_{j+1}$. Since the combined residue is $-\frac{1}{2}+1-\frac{1}{2}=0$, it follows that

$$
\int_{A_{j}} \phi^{\#}\left(\omega_{P}^{(\nu)}\right)=\overline{\int_{A_{j}} \omega_{P}^{(\nu)}}=0 .
$$

Therefore $\phi^{\#}\left(\omega_{P}^{(\nu)}\right)$ has all the properties that characterize $\omega_{P}^{(\nu)}$ and the lemma follows.

\subsection{The map $\Psi^{(\nu)}$ from $\left(P_{1}, \ldots, P_{N-1}\right)$ to the $B$-periods}

The differential $\omega_{P}^{(\nu)}$ has a vector of $B$-periods and it is convenient for us to divide by $2 \pi i$. So we define $\left(\beta_{1}, \ldots, \beta_{N-1}\right)$ with

$$
\beta_{k}=\frac{1}{2 \pi i} \int_{B_{k}} \omega_{P}^{(\nu)}
$$

Then by mapping the points $\left(P_{1}, \ldots, P_{N-1}\right)$ to the $B$-periods of the differential $\omega_{P}^{(\nu)}$, we obtain a map from $\Gamma_{1} \times \cdots \times \Gamma_{N-1}$ into $\mathbb{C}^{N-1}$. We will show that this map is well-defined from $\Gamma_{1} \times \cdots \times \Gamma_{N-1}$ to $(\mathbb{R} / \mathbb{Z})^{N-1}$. 
Proposition 2.3. For $\nu=1,2$, the map

$$
\begin{aligned}
& \Psi^{(\nu)}: \Gamma_{1} \times \cdots \times \Gamma_{N-1} \rightarrow(\mathbb{R} / \mathbb{Z})^{N-1}: \\
& \quad\left(P_{1}, \ldots, P_{N-1}\right) \mapsto\left(\beta_{1}, \ldots, \beta_{N-1}\right)=\frac{1}{2 \pi i}\left(\int_{B_{1}} \omega_{P}^{(\nu)}, \ldots, \int_{B_{N-1}} \omega_{P}^{(\nu)}\right)
\end{aligned}
$$

is well-defined and continuous.

Proof. Since the meromorphic differential $\omega_{P}^{(\nu)}$ depends continuously on the $P_{j}$ 's, we then also have that the $\beta_{k}$ from (2.8) is well-defined and continuous in the $P_{j}$ 's, unless $P_{k}$ is on $B_{k}$.

Recall that $B_{k}$ intersects the interval $\left(a_{k}, b_{k+1}\right)$ in one point on the first sheet. The value of $\beta_{k}$ then makes a jump when $P_{k} \in \Gamma_{k}$ passes through this intersection point. As the residue of $\omega_{P}^{(\nu)}$ at the pole $P_{k}$ is an integer (in fact, it is 1 , see (2.4) ), the jump in $\beta_{k}$ is by an integer value. Since we consider values modulo $\mathbb{Z}$, the map $\Psi^{(\nu)}$ is thus well-defined and continuous from $\Gamma_{1} \times \cdots \times \Gamma_{N-1}$ into $(\mathbb{C} / \mathbb{Z})^{N-1}$.

Now let us show that the $\beta_{k}$ are real and hence $\Psi^{(\nu)}$ is really a map into $(\mathbb{R} / \mathbb{Z})^{N-1}$. Recall that the cycle $B_{k}$ is chosen to be symmetric with respect to the real axis. Therefore $\phi\left(B_{k}\right)=-B_{k}$. Since $\omega_{P}^{(\nu)}=\phi^{\#}\left(\omega_{P}^{(\nu)}\right)$ by Lemma 2.2, we then have

$$
\int_{B_{k}} \omega_{P}^{(\nu)}=\int_{B_{k}} \phi^{\#}\left(\omega_{P}^{(\nu)}\right)=\overline{\int_{\phi\left(B_{k}\right)} \omega_{P}^{(\nu)}}=\overline{\int_{-B_{k}} \omega_{P}^{(\nu)}}=-\overline{\int_{B_{k}} \omega_{P}^{(\nu)}} .
$$

Thus $\int_{B_{k}} \omega_{P}^{(\nu)}$ is purely imaginary and so $\beta_{k}$ is real indeed.

The main result of this section is that the map (2.9) is a bijection. This would imply that there exists a unique set of points $\left(P_{1}^{(\nu)}, \ldots, P_{N-1}^{(\nu)}\right)$ such that the $B$-periods of the differential $\omega_{P}^{(\nu)}$ are given by $2 \pi i n \alpha_{j}$. The bijectivity proof relies on the fact that the divisor corresponding to any choice of points $P_{1}, \ldots, P_{N-1}$ with $P_{j} \in \Gamma_{j}$ is non-special.

We use additive notation for divisors and we write

$$
D=\sum_{j=1}^{N-1} P_{j}
$$

A divisor (2.10) is special if there exists a non-constant holomorphic function on $\mathcal{R} \backslash\left\{P_{1}, \ldots, P_{N-1}\right\}$ with at most simple poles at the points $P_{j}$. By the 
Riemann-Roch theorem, the divisor (2.10) is special if and only if there exists a non-zero holomorphic differential with zeros at each of the points $P_{j}$ for $j=1, \ldots, N-1$.

Lemma 2.4. (see also Statement 1 in [18]) If $P_{j} \in \Gamma_{j}$ for each $j=1, \ldots, N-$ 1 , then the divisor (2.10) is non-special.

Proof. The holomorphic differentials on the hyperelliptic Riemann surface defined by (2.1) are of the form

$$
\frac{p(z)}{w} d z
$$

where $p$ is a polynomial of degree $\leq N-2$. Therefore the zeros of a non-zero holomorphic differential project onto at most $N-2$ points in the complex $z$-plane. The points $P_{j} \in \Gamma_{j}, j=1, \ldots, N-1$, project onto $N-1$ distinct points, and so there can be no non-zero holomorphic differential with a zero at each of the $P_{j}$ 's.

In the proof of Theorem 2.6, which is the main result of this section, we also need the invariance of domain theorem of Brouwer 4, which is a classical result from topology. See e.g. [10, section XVII 3] or [14] for more recent accounts. We state the theorem here for the reader's convenience.

Theorem 2.5. (invariance of domain) If $U$ is an open subset of $\mathbb{R}^{n}$ and $f: U \rightarrow \mathbb{R}^{n}$ is an injective continuous map, then $f$ is open (i.e., $f$ maps open subsets of $U$ to open subsets of $\mathbb{R}^{n}$ ).

Of course, the theorem readily extends to injective continuous maps between manifolds of the same dimension, which is what we will use in the proof of Theorem 2.6.

Theorem 2.6. The map $\Psi^{(\nu)}$ defined in (2.9) is a bijection from $\Gamma_{1} \times \cdots \times$ $\Gamma_{N-1}$ to $(\mathbb{R} / \mathbb{Z})^{N-1}$.

Proof. We first prove that $\Psi^{(\nu)}$ is injective. Suppose $\left(P_{1}, \ldots, P_{N-1}\right)$ and $\left(Q_{1}, \ldots, Q_{N-1}\right)$ are in $\Gamma_{1} \times \cdots \times \Gamma_{N-1}$ so that

$$
\Psi^{(\nu)}\left(P_{1}, \ldots, P_{N-1}\right)=\Psi^{(\nu)}\left(Q_{1}, \ldots, Q_{N-1}\right) .
$$

Let $\omega_{P}^{(\nu)}$ and $\omega_{Q}^{(\nu)}$ be the corresponding meromorphic differentials. Then $\omega_{Q}^{(\nu)}-\omega_{P}^{(\nu)}$ has poles with residues \pm 1 at the points $Q_{j}$ and $P_{j}$ only and 
all periods are integer multiples of $2 \pi i$. Let $P_{0}$ be a given base point different from any of the $P_{j}$ 's and $Q_{j}$ 's. It then follows that

$$
\exp \left(\int_{P_{0}}^{z}\left(\omega_{Q}^{(\nu)}-\omega_{P}^{(\nu)}\right)\right), \quad z \in \mathcal{R}
$$

is a meromorphic function on $\mathcal{R}$ with only possible poles at $P_{1}, \ldots, P_{N-1}$. Since the divisor $D=\sum_{j=1}^{N-1} P_{j}$ is non-special, see Lemma 2.4, the meromorphic function is a constant, which implies that $\omega_{Q}^{(\nu)}=\omega_{P}^{(\nu)}$. Hence the $Q_{j}$ 's and the $P_{j}$ 's coincide: $\left(Q_{1}, \ldots, Q_{N-1}\right)=\left(P_{1}, \ldots, P_{N-1}\right)$ and therefore $\Psi^{(\nu)}$ is injective.

To prove surjectivity we now note that $\Psi^{(\nu)}$ is an injective and continuous (by Proposition 2.3) map from the $N-1$-dimensional manifold $\Gamma_{1} \times \cdots \times$ $\Gamma_{N-1}$ to the $N-1$-dimensional manifold $(\mathbb{R} / \mathbb{Z})^{N-1}$. Thus $\Psi^{(\nu)}$ is open by Theorem 2.5. It follows that $\Psi^{(\nu)}\left(\Gamma_{1} \times \cdots \times \Gamma_{N-1}\right)$ is a subset of $(\mathbb{R} / \mathbb{Z})^{N-1}$ that is both open (since $\Psi^{(\nu)}$ is open) and compact (since $\Psi^{(\nu)}$ is continuous and $\Gamma_{1} \times \cdots \times \Gamma_{N-1}$ is compact). Since $(\mathbb{R} / \mathbb{Z})^{N-1}$ is connected it follows that $\Psi^{(\nu)}$ is surjective.

\section{Construction of $M$}

It will be a consequence of Theorem 2.6 that we can construct the matrix $M$ that solves the model Riemann-Hilbert problem. that

By Theorem 2.6 there exist $P_{j}^{(\nu)} \in \Gamma_{j}$ for $j=1, \ldots, N-1, \nu=1,2$, so

$$
\Psi^{(\nu)}\left(P_{1}^{(\nu)}, \ldots, P_{N-1}^{(\nu)}\right)=\left(n \alpha_{1}, \ldots, n \alpha_{N-1}\right)
$$

where each $n \alpha_{j}$ is considered modulo $\mathbb{Z}$. Let $\omega_{P}^{(\nu)}$ be the corresponding meromorphic differential. We then have that

$$
\frac{1}{2 \pi i} \int_{B_{k}} \omega_{P}^{(\nu)} \equiv n \alpha_{k} \quad \bmod \mathbb{Z}, \quad \text { for } k=1, \ldots, N-1 .
$$

\subsection{Abelian integrals}

For $\nu=1,2$, we choose the base point

$$
P_{0}=\infty_{\nu}
$$

and we define the functions $u_{1}^{(\nu)}(z), u_{2}^{(\nu)}(z)$ of a complex variable $z$ as follows. 
Definition 3.1. Let $z \in \mathbb{C} \backslash \mathbb{R}$.

(a) To define $u_{j}^{(\nu)}$ with $j=\nu$, we consider $z$ as a point on the $j$ th sheet of the Riemann surface. We define

$$
u_{j}^{(\nu)}(z)=\int_{P_{0}}^{z} \omega_{P}^{(\nu)}, \quad z \in \mathbb{C} \backslash \mathbb{R}, \quad j=\nu,
$$

where the path of integration is on the $j$ th sheet of the Riemann surface and it does not intersect the real line, except for the initial point $P_{0}$.

(b) To define $u_{j}^{(\nu)}(z)$ with $j \neq \nu$, we consider $z$ as a point on the $j$ th sheet. We define

$$
u_{j}^{(\nu)}(z)=\int_{P_{0}}^{z} \omega_{P}^{(\nu)}, \quad z \in \mathbb{C} \backslash \mathbb{R}, \quad j \neq \nu,
$$

where now the path of integration is as follows. If $\operatorname{Im} z>0(\operatorname{Im} z<0)$ then the path starts in the lower (upper) half-plane of the $\nu$ th sheet and passes to the $j$ th sheet via a cut $\left(a_{k}, b_{k}\right)$. It then stays in the upper (lower) half-plane of the $j$ th sheet.

Since $\omega_{P}^{(\nu)}$ has vanishing $A$-periods, as well as vanishing $\phi^{\#}(A)$ periods, it does not matter which cut $\left(a_{k}, b_{k}\right)$ is taken, and so $u_{j}^{(\nu)}(z)$ in (3.5) is uniquely defined.

The functions $u_{j}^{(\nu)}$ are analytic on $\mathbb{C} \backslash \mathbb{R}$ with the following jumps on $\mathbb{R}$.

Lemma 3.2. The functions $u_{j}^{(\nu)}, j, \nu=1,2$ satisfy the following jump conditions for $x \in \mathbb{R}$.

(a) For $x \in\left(a_{k}, b_{k}\right)$ with $k=1, \ldots, N$, we have

$$
\begin{aligned}
& u_{1,+}^{(\nu)}(x)=u_{2,-}^{(\nu)}(x), \\
& u_{2,+}^{(\nu)}(x)=u_{1,-}^{(\nu)}(x) .
\end{aligned}
$$

(b) For $x<a_{1}$ or $x>b_{N}$ we have

$$
\begin{aligned}
& u_{j,+}^{(\nu)}(x)=u_{j,-}^{(\nu)}(x), \quad j=\nu, \\
& u_{j,+}^{(\nu)}(x) \equiv u_{j,-}^{(\nu)}(x)+\pi i \quad \bmod 2 \pi i \mathbb{Z}, \quad j \neq \nu .
\end{aligned}
$$


(c) For $z \in \mathbb{C} \backslash \bigcup_{k=1}^{N}\left[a_{k}, b_{k}\right]$, we use $P_{j}(z)$ to denote the point on the $j$ th sheet of $\mathcal{R}$ that corresponds to $z$. Then we have, for $x \in\left(b_{k}, a_{k+1}\right)$ with $k=1, \ldots, N-1$,

$$
\begin{array}{lrr}
u_{1,+}^{(1)}(x) & \equiv u_{1,-}^{(1)}(x)-2 \pi i n \alpha_{k}, & \text { if } P_{1}(x) \neq P_{k}^{(1)}, \\
u_{2,+}^{(1)}(x) \equiv u_{2,-}^{(1)}(x)+2 \pi i n \alpha_{k}+\pi i & \text { if } P_{2}(x) \neq P_{k}^{(1)}, \\
u_{1,+}^{(2)}(x) \equiv u_{1,-}^{(2)}(x)-2 \pi i n \alpha_{k}+\pi i & \text { if } P_{1}(x) \neq P_{k}^{(2)}, \\
u_{2,+}^{(2)}(x) \equiv u_{2,-}^{(2)}(x)+2 \pi i n \alpha_{k}, & \text { if } P_{2}(x) \neq P_{k}^{(2)},
\end{array}
$$

The equalities (3.10) $-(3.13)$ are valid modulo $2 \pi i \mathbb{Z}$.

Proof. The properties (3.6) and (3.7) follow immediately from the definition of $u_{j}^{(\nu)}$.

Let $\Delta^{(\nu)}$ be the set of poles for $\omega_{P}^{(\nu)}$, that is,

$$
\Delta^{(\nu)}=\left\{a_{1}, b_{1}, \ldots, a_{N}, b_{N}, P_{1}^{(\nu)}, \ldots, P_{N-1}^{(\nu)}, \infty_{j}\right\}, \quad j \neq \nu .
$$

For $x<a_{1}$ and $x>b_{N}$, we have

$$
u_{j,+}^{(\nu)}(x)-u_{j,-}^{(\nu)}(x)=\oint_{C} \omega_{P}^{(\nu)}
$$

where $C$ is a closed contour on $\mathcal{R} \backslash \Delta^{(\nu)}$. For $j=\nu$, the contour $C$ is contractible in $\mathcal{R} \backslash \Delta^{(\nu)}$ and (3.8) follows. When $j \neq \nu$, we choose the contour to pass through the cut $\left[a_{1}, b_{1}\right]$ in case $x<a_{1}$, and through the cut $\left[a_{N}, b_{N}\right]$ in case $x>b_{N}$. Then $C$ is contractible to a small loop around $a_{1}$ (in case $x<a_{1}$ ) or around $b_{N}$ (in case $x>b_{N}$ ). Since the residues of $\omega_{P}^{(\nu)}$ at $a_{1}$ and $b_{N}$ are $-\frac{1}{2}$ we have in either case

$$
\oint_{C} \omega_{P}^{(\nu)}=\pi i \quad \bmod 2 \pi i,
$$

and (3.9) follows.

Let $x \in\left(b_{k}, a_{k+1}\right), x \neq z\left(P_{k}^{(\nu)}\right)$, for some $k=1, \ldots, N-1$. Then we have

$$
u_{j,+}^{(\nu)}(x)-u_{j,-}^{(\nu)}(x)=\oint_{C} \omega_{P}^{(\nu)}
$$

where $C$ is again a closed contour on $\mathcal{R} \backslash \Delta^{(\nu)}$. When $j=\nu=1$, then $C$ is on the first sheet and can be deformed into $-B_{k}$ and (3.10) follows because of (3.2). 
When $j=\nu=2$, then $C$ is a closed contour on the second sheet, and it is homotopic to $B_{k}$ in $\mathcal{R}$, but the deformation will pick up residue contributions from the poles at $a_{1}, \ldots, a_{k}, b_{1}, \ldots, b_{k}, P_{1}^{(\nu)}, \ldots, P_{k-1}^{(\nu)}$ and possibly $P_{k}^{(\nu)}$ depending on its position. Since the combined residues of $\omega_{P}^{(\nu)}$ at these poles is an integer number, the poles do not contribute (modulo $2 \pi i$ ) and we obtain (3.13) from (3.2).

When $j \neq \nu$, the closed loop $C$ is on both sheets, For $j=2, \nu=1$, we choose $C$ so that it passes through the cut $\left[a_{k}, b_{k}\right]$. Then $C$ is homotopic to $B_{k}$ in $\mathcal{R}$, but a deformation from $C$ to $B_{k}$ will pick up a residue contribution from $b_{k}$ and possibly from $P_{k}^{(\nu)}$. The combined residue is $-1 / 2$ or $+1 / 2$, and this leads to (3.11).

Finally, for $j=1, \nu=2$, we choose $C$ so that it passes through the cut $\left[a_{1}, b_{1}\right]$. Then $C$ is homotopic to $-B_{k}$ in $\mathcal{R}$, and the deformation from $C$ to $-B_{k}$ picks up a residue contribution at $a_{1}$ and possibly $P_{k}^{(\nu)}$. The combined residue is $-1 / 2$ or $+1 / 2$, and we obtain (3.12).

Note that in part (c) of Lemma 3.2 we excluded the case $P_{j}(x)=P_{k}^{(\nu)}$ since $P_{k}^{(\nu)}$ is a pole of $\omega_{P}^{(\nu)}$ and so the limiting values $u_{j,+}^{(\nu)}(x)$ and $u_{j,-}^{(\nu)}(x)$ do not exist if $P_{k}^{(\nu)}$ is on the $j$ th sheet, see also part (b) of Lemma 3.3.

The behavior near all poles of $\omega_{P}^{(\nu)}$ is stated in the following lemma. We use $z(P)$ to denote the $z$-coordinate of a point $P=(z, w)$ on the Riemann surface (2.1).

Lemma 3.3. We have

(a) for $j, \nu=1,2$ and $k=1, \ldots, N$,

$$
\begin{aligned}
u_{j}^{(\nu)}(z) & =-\frac{1}{4} \log \left(z-a_{k}\right)+O(1), \quad \text { as } z \rightarrow a_{k}, \\
u_{j}^{(\nu)}(z) & =-\frac{1}{4} \log \left(z-b_{k}\right)+O(1), \quad \text { as } z \rightarrow b_{k},
\end{aligned}
$$

(b) if $P_{k}^{(\nu)}$ is on the $j$ th sheet of the Riemann surface, then

$$
u_{j}^{(\nu)}(z)=\log \left(z-z\left(P_{k}^{(\nu)}\right)\right)+O(1) \quad \text { as } z \rightarrow z\left(P_{k}^{(\nu)}\right),
$$

(c) as $z \rightarrow \infty$

$$
\begin{aligned}
& u_{j}^{(\nu)}(z)=O(1 / z), \quad \text { if } j=\nu \\
& u_{j}^{(\nu)}(z)=-\log z+O(1), \quad \text { if } j \neq \nu .
\end{aligned}
$$


Proof. The fact that $u_{j}^{(\nu)}(z)=O(1 / z)$ as $z \rightarrow \infty$ in case $j=\nu$ follows directly from (3.4) since $P_{0}=\infty_{\nu}$. The other statements of the lemma follow from (3.4) $-(3.5)$ and the residue conditions in (2.3) $-(2.5)$.

In Lemma 3.3 we implicitly assumed that the point $P_{k}^{(\nu)}$ is different from $b_{k}$ and $a_{k+1}$. If for example, $P_{k}^{(\nu)}=b_{k}$, then the residue of $\omega_{P}^{(\nu)}$ at $b_{k}$ is equal to $+\frac{1}{2}$, and in part (a) of Lemma 3.3 we get

$$
u_{j}^{(\nu)}(z)=\frac{1}{4} \log \left(z-b_{k}\right)+O(1), \quad \text { as } z \rightarrow b_{k} .
$$

The modifications that are needed when one or more of the $P_{k}^{(\nu)}$ coincide with an endpoint are obvious, and we will not specify them in the rest of the paper.

\subsection{Exponential of the Abelian integrals}

Now we exponentiate the functions $u_{j}^{(\nu)}$.

Definition 3.4. We define $v_{j}^{(\nu)}, j, \nu=1,2$ by

$$
v_{j}^{(\nu)}(z)=\exp \left(u_{j}^{(\nu)}(z)\right), \quad z \in \mathbb{C} \backslash \mathbb{R} .
$$

Then the functions $v_{j}^{(\nu)}(z)$ are analytic in $\mathbb{C} \backslash \mathbb{R}$ with the following jumps on $\mathbb{R}$.

Corollary 3.5. The vectors $\left(v_{1}^{(\nu)}, v_{2}^{(\nu)}\right)$ satisfy the following jump conditions on $\mathbb{R} \backslash\left\{a_{1}, b_{1}, \ldots, a_{N}, b_{N}\right\}$,

$$
\left(v_{1}^{(\nu)}, v_{2}^{(\nu)}\right)_{+}=\left(v_{1}^{(\nu)}, v_{2}^{(\nu)}\right)_{-} J_{v}^{(\nu)}, \quad \nu=1,2,
$$

where

$$
\begin{aligned}
& J_{v}^{(\nu)}(x)=\left(\begin{array}{ll}
0 & 1 \\
1 & 0
\end{array}\right) \quad \text { for } a_{k}<x<b_{k}, \quad k=1, \ldots, N, \\
& J_{v}^{(\nu)}(x)=(-1)^{\nu-1}\left(\begin{array}{cc}
1 & 0 \\
0 & -1
\end{array}\right) \quad \text { for } x<a_{1} \text { or } x>b_{N}, \\
& J_{v}^{(\nu)}(x)=(-1)^{\nu-1}\left(\begin{array}{cc}
e^{-2 \pi i n \alpha_{k}} & 0 \\
0 & -e^{2 \pi i n \alpha_{k}}
\end{array}\right) \quad \text { for } b_{k}<x<a_{k+1},
\end{aligned}
$$

for $k=1, \ldots, N-1$. 
Proof. The jumps follow directly from Lemma 3.2 and Definition 3.4 in case $x \neq z\left(P_{k}^{(\nu)}\right)$.

If $x=z\left(P_{k}^{(\nu)}\right)$ and $P_{k}^{(\nu)}$ is on the $j$ th sheet, then Lemma 3.2 does not apply to $u_{j}$. However, in that case we find by part (b) of Lemma 3.3 and Definition 3.4 that

$$
v_{j,+}^{(\nu)}(x)=v_{j,-}^{(\nu)}(x)=0,
$$

and (3.16), (3.19) is also valid.

From Lemma 3.3 and (3.15) we find the following behavior near the poles of $\omega_{P}^{(\nu)}$.

Corollary 3.6. We have

(a) for $j, \nu=1,2$ and $k=1, \ldots, N$,

$$
\begin{array}{ll}
v_{j}^{(\nu)}(z)=O\left(\left(z-a_{k}\right)^{-1 / 4}\right), & \text { as } z \rightarrow a_{k}, \\
v_{j}^{(\nu)}(z)=O\left(\left(z-b_{k}\right)^{-1 / 4}\right), & \text { as } z \rightarrow b_{k}
\end{array}
$$

(b) if $P_{k}^{(\nu)}$ is on the $j$ th sheet of $\mathcal{R}$, then

$$
v_{j}^{(\nu)}(z)=O\left(z-z\left(P_{k}^{(\nu)}\right)\right) \quad \text { as } z \rightarrow z\left(P_{k}^{(\nu)}\right)
$$

(c) as $z \rightarrow \infty$

$$
\begin{aligned}
& v_{j}^{(\nu)}(z)=1+O(1 / z), \quad \text { if } j=\nu \\
& v_{j}^{(\nu)}(z)=O(1 / z), \quad \text { if } j \neq \nu .
\end{aligned}
$$

Note that by (3.20) the function $v_{j}^{(\nu)}$, has a zero at $z\left(P_{k}^{\nu}\right)$ in case $P_{k}^{(\nu)}$ is on the $j$ th sheet.

\subsection{The parametrix $M$}

To construct the solution $M$ of the model Riemann-Hilbert problem in Definition 1.1, we only need a trivial modification of the functions $v_{j}^{(\nu)}$. 
Definition 3.7. We define

$$
M(z)= \begin{cases}\left(\begin{array}{cc}
v_{1}^{(1)}(z) & v_{2}^{(1)}(z) \\
-v_{1}^{(2)}(z) & v_{2}^{(2)}(z)
\end{array}\right), & \text { if } \operatorname{Im} z>0 \\
\left(\begin{array}{cc}
v_{1}^{(1)}(z) & -v_{2}^{(1)}(z) \\
v_{1}^{(2)}(z) & v_{2}^{(2)}(z)
\end{array}\right), & \text { if } \operatorname{Im} z<0 .\end{cases}
$$

From (3.16)-(3.19), it is easy to verify that $M(z)$ does indeed satisfy the jump conditions (1.1)-(1.2) of the RH problem in Definition 1.1. The asymptotic condition $M(z)=I+O(1 / z)$ as $z \rightarrow \infty$ holds because of part (c) of Corollary 3.6. Part (a) of Corollary 3.6 shows that $M$ has at most fourth root singularities at the endpoints $a_{k}$ and $b_{k}$. So we have proved the main result of this paper:

Theorem 3.8. The matrix-valued function $M(z)$ defined by (3.21) satisfies the model Riemann-Hilbert problem of Definition 1.1.

\section{Properties of $M$}

We collect here some further properties of $M$ that are useful in applications.

\subsection{Uniqueness of the solution}

The first two properties are standard, see e.g. [5]

Proposition 4.1. For every $z \in \mathbb{C} \backslash\left[a_{1}, b_{N}\right]$ we have

$$
\operatorname{det} M(z)=1 \text {. }
$$

Proof. Since the jump matrices in the model Riemann-Hilbert have determinant 1 , the function $z \mapsto \operatorname{det} M(z)$ has no jump discontinuities in $\mathbb{C}$, and so it has an analytic extension to $\mathbb{C} \backslash\left\{a_{1}, b_{1}, \ldots, a_{N}, b_{N}\right\}$. From part (d) in Definition 1.1, we conclude that $\operatorname{det} M(z)$ can have at most square-root singularities at the endpoint $a_{j}, b_{j}$, and therefore these isolated singularties are removable. Thus $z \mapsto \operatorname{det} M(z)$ is an entire function, which by the asymptotic condition satisfies $\operatorname{det} M(z)=1+O\left(z^{-1}\right)$ as $z \rightarrow \infty$. Then the proposition follows, by Liouville's theorem. 
Proposition 4.2. The solution $M$ of the model RH problem is unique.

Proof. Let $\widetilde{M}$ be a second solution of the model RH problem. By Proposition 4.1, we have that $M(z)$ is invertible for every $z \in \mathbb{C} \backslash\left[a_{1}, b_{N}\right]$. Then

$$
H(z)=\widetilde{M}(z) M(z)^{-1}, \quad z \in \mathbb{C} \backslash\left[a_{1}, b_{N}\right]
$$

is well-defined and analytic. Since $\widetilde{M}$ and $M$ satisfy the same jump conditions, it follows that $H_{+}(x)=H_{-}(x)$ for every $x \in\left[a_{1}, b_{N}\right] \backslash\left\{a_{1}, b_{1}, \ldots, a_{N}, b_{N}\right\}$. The entries of $\widetilde{M}$ and $M^{-1}$ have at most fourth root singularities at the endpoints $a_{j}, b_{j}$. Thus $H$ has at most square root singularities, and it follows that the singularities are removable. Thus $H$ has an extension to an entire function. Since $H(z)=I+O(1 / z)$ as $z \rightarrow \infty$, we find by Liouville's theorem that $H(z)=I$ for every $z \in \mathbb{C}$. The proposition follows.

\subsection{Zeros}

By (3.4) and (3.21) the entries of $M(z)$ do not vanish at any point $z \in$ $\mathbb{C} \backslash\left[a_{1}, b_{N}\right]$. Across each cut $\left(a_{k}, b_{k}\right), k=1, \ldots, N$, and each gap $\left(b_{k}, a_{k+1}\right)$, $k=1, \ldots, N-1$ the entries have an analytic continuation given by the $\mathrm{RH}$ problem. By (3.20) we have that $v_{j}^{(\nu)}(z) \rightarrow 0$ as $z \rightarrow z\left(P_{k}^{(\nu)}\right)$ if $P_{k}^{(\nu)}$ is on the $j$ th sheet. By (3.21) this translates into the following statement about the zeroes of the entry $M_{\nu, j}$ of $M$.

Proposition 4.3. If $P_{k}^{(\nu)}$ is on the $j$ th sheet then $M_{\nu, j}(z)$ has a simple zero at $z=z\left(P_{k}^{(\nu)}\right)$, in the sense that the restriction of $M_{\nu, j}$ to the upper (lower) halfplane has an analytic continuation across $\left(b_{k}, a_{k+1}\right)$ into the lower (upper) half-plane which has a simple zero at $z=z\left(P_{k}^{(\nu)}\right)$.

The points $z=z\left(P_{k}^{(\nu)}\right)$ are the only possible zeros of the (analytic continuations of the) entries of $M$.

Proof. Everything is already proved, except for the fact that the zero is simple. This follows from the fact that $M_{\nu, j}= \pm e^{u_{j}^{(\nu)}}$ where $u_{j}^{(\nu)}(z)$ has the behavior (3.14) as $z \rightarrow z\left(P_{k}^{(\nu)}\right)$.

\subsection{Uniform boundedness}

The solution $M$ clearly depends on $n$. The following uniform boundedness property is needed in the construction of a local parametrix in the steepest descent analysis, see [7]. 
Proposition 4.4. For every $\varepsilon>0$, we have that $M(z)$ and $M^{-1}(z)$ are uniformly bounded in $n$ for $z$ in the set

$$
\left\{z \in \mathbb{C} \backslash\left[a_{1}, b_{N}\right]|| z-a_{j}|\geq \varepsilon,| z-b_{j} \mid \geq \varepsilon \text { for all } j=1, \ldots, N\right\} .
$$

Proof. It follows easily from our construction that for every $\left(\beta_{1}, \ldots, \beta_{N-1}\right) \in$ $(\mathbb{R} / \mathbb{Z})^{N-1}$ there is a unique solution to the model $\mathrm{RH}$ problem where the jump on $\left(a_{j}, b_{j+1}\right)$ is replaced by

$$
M_{+}(x)=M_{-}(x)\left(\begin{array}{cc}
e^{-2 \pi i \beta_{j}} & 0 \\
0 & e^{2 \pi i \beta_{j}}
\end{array}\right), \quad x \in\left(a_{j}, b_{j+1}\right)
$$

for $j=1, \ldots, N-1$. If we denote the solution of this $\mathrm{RH}$ problem by

$$
M\left(z ; \beta_{1}, \ldots, \beta_{N-1}\right)
$$

then the solutions we are interested in are

$$
M\left(z ; n \alpha_{1}, \ldots, n \alpha_{N-1}\right), \quad n \in \mathbb{N},
$$

and so they are part of this family (4.2). It is therefore enough to show that the solutions (4.2) are uniformly bounded on the set (4.1).

The map

$$
\left(\beta_{1}, \ldots, \beta_{N-1}\right) \mapsto M\left(z ; \beta_{1}, \ldots, \beta_{N-1}\right)
$$

is continuous as a map from $(\mathbb{R} / \mathbb{Z})^{N-1}$ to the $2 \times 2$-matrix valued analytic functions on $\mathbb{C} \backslash\left[a_{1}, b_{N}\right]$ provided with the topology of uniform convergence on compact subsets of $\mathbb{C} \backslash\left\{a_{1}, b_{1}, \ldots, a_{N}, b_{N}\right\}$. Since $(\mathbb{R} / \mathbb{Z})^{N-1}$ is compact, we then have that the functions $M\left(z ; \beta_{1}, \ldots, \beta_{N-1}\right)$ are also compact in this space, which in particular implies that the functions are uniformly bounded on every set of the form (4.1). So $M$ is uniformly bounded in $n$ on (4.1).

Since $\operatorname{det} M \equiv 1$, the entries of $M^{-1}$ are, up to a sign, the same as those of $M$, and so $M^{-1}$ is also uniformly bounded in $n$ on (4.1).

\section{Alternative construction for the second row}

The above construction of the solution $M$ of the model $\mathrm{RH}$ problem is done row by row. Indeed, the case $\nu=1$ leads to the first row, and the case $\nu=2$ leads to the second row of $M$. The difference between the two cases lies in the condition (2.5) that specifies which point at infinity is a pole of 
the meromorphic differential. Otherwise the two cases are similar, and we treated them simultaneously.

The fact that these two cases are similar is also related to the hyperelliptic Riemann surface, which possesses hyperelliptic involution interchanging the sheets. In other situations related to multi-sheeted Riemann surfaces as in [11], 21], there is no simple symmetry between the sheets. In addition, the point at infinity is a branch point in [11, [21] that connects all but one of the sheets. For the construction of a model $\mathrm{RH}$ problem in such situations, it may be of interest to realize that the construction of one row of the model $\mathrm{RH}$ problem can help to construct the other rows.

We illustrate this here for the hyperelliptic case, and so we give an alternative way to construct the second row, on the assumption that we know the first row of $M$. We thank Alexander Aptekarev for this remark.

Recall that the construction of the first row of $M$ is based on the points $P_{k}^{(1)} \in \Gamma_{k}, k=1, \ldots, N-1$ satisfying (3.1). In Lemma 2.4 we proved that the divisor

$$
D=\sum_{k=1}^{N-1} P_{k}^{(1)}
$$

is non-special.

Lemma 5.1. The vector space of meromorphic functions on $\mathcal{R}$ (including constant functions) with divisor greater than or equal to $-\sum_{k=1}^{N-1} P_{k}^{(1)}-\infty_{2}$ is of dimension 2.

Proof. Let us denote, for a positive divisor $D^{\prime}$, the space of meromorphic functions on $\mathcal{R}$ whose divisor is greater than or equal to $-D^{\prime}$ by $L\left(D^{\prime}\right)$.

Since $D=\sum_{k=1}^{N-1} P_{k}^{(1)}$ is non-special, the only functions in $L(D)$ are constant functions, so that

$$
\operatorname{dim} L(D)=1 \text {. }
$$

Also, $L(D)$ is the kernel of the linear functional from $L\left(D+\infty_{2}\right)$ to $\mathbb{C}$ that maps a function in $L\left(D+\infty_{2}\right)$ to its residue at $\infty_{2}$. Thus by the dimension theorem for linear functionals,

$$
\operatorname{dim} L\left(D+\infty_{2}\right) \leq \operatorname{dim} L(D)+1=2 .
$$

On the other hand, the divisor $D+\infty_{2}$ is of degree $N$, so that by the Riemann-Roch theorem, see [13],

$$
\operatorname{dim} L\left(D+\infty_{2}\right) \geq 2
$$


This proves the lemma.

Suppose now that we have the first row of $M$. We can then construct the second row of $M$ by modifying the first row $\left(M_{11}, M_{12}\right)$ by a meromorphic factor as follows.

By Lemma 5.1, the space of meromorphic functions $F$ on $\mathcal{R}$ with divisor $\geq-\sum_{k=1}^{N-1} P_{k}^{(1)}-\infty_{2}$ is two-dimensional. The following two conditions on $F$ (recall that $M_{12}$ is analytic in a neighborhood of $\infty_{2}$ with a simple zero at $\left.\infty_{2}\right)$

$$
F\left(\infty_{1}\right)=0, \quad \text { and } \quad \lim _{z \rightarrow \infty_{2}} M_{12}(z) F(z)=1,
$$

determine $F$ uniquely.

We use $F_{1}$ and $F_{2}$ to denote the restrictions of $F$ to the first and second sheet, respectively. Then the two functions defined by

$$
M_{21}(z)=M_{11}(z) F_{1}(z), \quad M_{22}(z)=M_{12}(z) F_{2}(z)
$$

satisfy all conditions that we need for the entries in the second row of $M$. Note also that the poles of $F_{1}$ and $F_{2}$ at $P_{1}^{(1)}, \ldots, P_{N-1}^{(1)}, \infty_{2}$ are cancelled by the zeros of $M_{11}$ and $M_{12}$ at these points, see also Proposition 4.3,

\section{Acknowledgement}

We thank Alexander Aptekarev for helpful discussions.

\section{References}

[1] A.I. Aptekarev, Analysis of the matrix Riemann-Hilbert problems for the case of higher genus - asymptotics of polynomials orthogonal on a system of intervals, KIAM Preprint No 28, Keldysh Institute of Appl. Math., Moscow, 2008.

[2] P. Bleher, S. Delvaux, and A.B.J. Kuijlaars, Random matrix model with external source and a constrained vector equilibrium problem, to appear in Comm. Pure Appl. Math., Preprint arXiv:1001.1238.

[3] A.A. Bolibruch, The Riemann-Hilbert problem, Russian Math. Surveys 45 (2) (1990), 1-58. 
[4] L.E.J. Brouwer, Zur Invarianz des $n$-dimensionalen Gebiets, Math. Ann. 72 (1912), 55-56.

[5] P. Deift, Orthogonal Polynomials and Random Matrices: a RiemannHilbert approach. Courant Lecture Notes in Mathematics Vol. 3, Amer. Math. Soc., Providence R.I. 1999.

[6] P. Deift, A.R. Its and X. Zhou, A Riemann-Hilbert approach to asymptotic problems arising in the theory of random matrix models, and also in the theory of integrable statistical mechanics, Ann. of Math. 146 (1997), 149-235.

[7] P. Deift, T. Kriecherbauer, K.T-R McLaughlin, S. Venakides, and X. Zhou, Uniform asymptotics for polynomials orthogonal with respect to varying exponential weights and applications to universality questions in random matrix theory, Comm. Pure Appl. Math. 52 (1999), 1335-1425.

[8] P. Deift and X. Zhou. A steepest descent method for oscillatory Riemann-Hilbert problems. Asymptotics for the MKdV equation, Ann. of Math. (2) 137 (1993), no. 2, 295-368.

[9] P. Deift, A. Its, A. Kapaev, and X. Zhou, On the algebro-geometric integration of the Schlesinger equations, Comm. Math. Phys. 203 (1999), no. 3, 613-633.

[10] J. Dugundji, Topology, Allyn and Bacon, Inc., Boston, Mass., 1966.

[11] M. Duits and A.B.J. Kuijlaars, Universality in the two matrix model: a Riemann-Hilbert steepest descent analysis, Comm. Pure Appl. Math. 62 (2009), 1076-1153.

[12] M. Duits, A.B.J. Kuijlaars, and M.Y. Mo, The Hermitian two matrix model with an even quartic potential, in preparation.

[13] H. Farkas and I. Kra, Riemann Surfaces, Graduate Texts in Mathematics 71, Springer-Verlag, New York-Berlin, 1980.

[14] W. Fulton, Algebraic Topology, a First Course, Springer-Verlag, New York, 1995. 
[15] M. Jimbo and T. Miwa, Monodromy preserving deformation of linear ordinary differential equations with rational coefficients II, Physica D 2 (1981), 407-448.

[16] S. Kamvissis, K.D.T-R McLaughlin, and P.D. Miller, Semiclassical soliton ensembles for the focusing nonlinear Schrdinger equation Ann. Math. Studies 154, Princeton University Press, Princeton, NJ, 2003.

[17] A. V. Kitaev and D. Korotkin, On solutions of the Schlesinger equations in terms of $\Theta$-functions, Internat. Math. Res. Notices (1998), 877-905.

[18] D. Korotkin, Introduction to the functions on compact Riemann surfaces and theta-functions, in "Nonlinearity and Geometry" (D. Wojcik and J. Cieslinski, eds.) Polish Scient. Publ. PWN, Warsaw, 1998, pp. 109-139. Preprint arXiv:solv-int/9911002

[19] D. Korotkin, Solution of matrix Riemann-Hilbert problems with quasipermutation monodromy matrices, Math. Ann. 329 (2004), 335-364.

[20] B. Malgrange, Sur les déformations isomonodromiques I, singularités régulières, in "Mathematics and Physics" (Paris, 1979/1982), Progress in Mathematics 37, Birkhäuser, Boston, 1983, pp. 401-426.

[21] M.Y. Mo, Universality in the two matrix model with a monomial quartic and a general even polynomial potential, Commun. Math. Phys. 291 (2009), 863-894.

[22] W. Van Assche, J. Geronimo, and A.B.J. Kuijlaars, Riemann-Hilbert problems for multiple orthogonal polynomials, in "Special Functions 2000: Current Perspectives and Future Directions" (J. Bustoz et al., eds.), Kluwer, Dordrecht, 2001, pp. 23-59. 